\title{
Sistema control supervisor de clientes con acceso remoto para sistemas solares fotovoltaicos autónomos
}

\section{supervisory control system for clients whit remote access of autonomous photovoltaic solar sistems}

\author{
Alberto Nicolás Figueroa-Cuello' \\ Aldo Pardo-García² \\ Jorge Luis Díaz-Rodríguez
}

Recibido: agosto 15 de 2016

Aceptado: diciembre 28 de 2016

\section{Resumen}

Los Sistemas Solares Fotovoltaicos Autónomos (SFVA), a pesar de sus ventajas, presentan algunos inconvenientes, siendo el principal de ellos la falta de un sistema controlador de energía para optimizar su funcionamiento. Este trabajo presenta el análisis y diseño de un Sistema de Control Supervisor de Acceso Remoto, SCSAR, para clientes con acceso remoto de sistemas solares fotovoltaicos autónomos de $500 \mathrm{~W}$. La función del SCSAR es la de medir y administrar la energía consumida por los usuarios del SFVA. Para ello se consideran tres variables principales: el tiempo de conexión y desconexión por carga y por día, autorizados al usuario; la corriente consumida por cada dispositivo o carga autorizado por el diseñador; el nivel de carga de la batería para mantenerlo por encima del mínimo permitido para su correcta operación. El SCSAR aquí propuesto, es un sistema que garantiza que se cumplan los objetivos de diseño, monitoreando y controlando las variables críticas de los sistemas clientes. De esta forma será posible diagnosticar remotamente los SFVA, reduciendo los tiempos y costos de soporte y mantenimiento, con el fin de brindar seguridad, disponibilidad, versatilidad, economía, robustez y confiabilidad a todos los usuarios de estas fuentes de energía alternativa.

Palabras clave: control supervisor, sistemas solares fotovoltaicos, SCADA, cliente-servidor, monitoreo.

\begin{abstract}
The Autonomous Photovoltaic Solar Systems (APSS), in spite of its disadvantages, offers some inconveniences, being the main one of them, the lack of an energy control system to optimize its operation. This work presents the analysis and design of a Remote Access Supervisor Control System, RASCS for customers with remote access of $500 \mathrm{~W}$ photovoltaic solar systems. The RASCS's function is to measure and manage the energy consumed by the users of APSS. For this reason, three main variables are considered: the time of connection and disconnection per load and per day, authorized to the user; the current consumed by each device or charger authorized by the designer; the battery charge level to keep it above the proper amount for proper operation. RASCS here is a system that ensures that it meets the design objectives, monitoring and controlling the critical variables of the customer systems. In this way, it will be possible to remotely diagnose APSS, reducing the time and costs of support and maintenance, with the completion of security, availability, versatility, economy, robustness and reliability to all users of these alternative energy sources.
\end{abstract}

Keywords: supervisory control, solar photovoltaic systems, APSS, SFVA, client-server, monitoring.

1 Ingeniero Electrónico, Magíster en Control Industrial, Servicio Nacional de Aprendizaje, Colombia. E-mail: anfcuello@gmail.com

2 Ingeniero eléctrico, Doctor en Complejos Eléctricos y Electrónicos, Universidad de Pamplona, Colombia. E-mail: apardo13@hotmail.com

3 Ingeniero Eléctrico, Máster en Automática, Universidad de Pamplona, Colombia. E-mail: jdiazcu@gmail.com 


\section{Introducción}

En los últimos años los sistemas solares fotovoltaicos han tenido un auge en su utilización en zonas aisladas, industriales, agropecuarias e incluso urbanas, como respuesta a la problemática del abastecimiento energético mundial y como fuente de energía amigable con el medio ambiente (Sánchez, \& Escobar, 2009; Sánchez-Molina, Gelves-Díaz, \& Ramírez, 2014; Reyes-Caballero, Fernández-Morales, \& Duarte, 2016). Esto se debe a que la energía solar es renovable, abundante, limpia, gratuita e inagotable con respecto al ciclo de vida humano (Beleño-Sáenz, Pardo-García, \& Torres-Chávez, 2013).

Adicionalmente, a diferencia de otros generadores de energía eléctrica, los equipos requeridos para la generación de la energía fotovoltáica no tienen partes móviles, son de tipo modular, fácilmente ampliables e incluso, en algunos casos, transportables, su mantenimiento es mínimo, tienen larga vida útil y no produce ningún ruido durante su operación (Beleño-Sáenz, TorresChávez, Pardo-García, \& Díaz-Rodríguez, 2011; Sandoval-Ruiz, 2014; García, 2005; Vargas, Velásquez-Clavijo, \& Torres-Gómez, 2016).

Lo más relevante es que para producir energía estos equipos no consumen combustibles fósiles, como: carbón, petróleo o gas natural (MartínezBernal, 2013; Gómez-Rojas, Díaz-Lagos, BlandónMontes, \& Martínez-Ovalle, 2016). Estos recursos, además de no ser renovables, emiten óxidos de nitrógeno y dióxido de carbono, causantes del calentamiento global, la lluvia ácida, el efecto invernadero, el esmog, generación de residuos nucleares, la contaminación del agua, la destrucción de hábitats por los derrames de petróleo y la pérdida de los recursos naturales (Leal-González, \& Hernández-Cely, 2014; VidesHerrera, Pardo-García, \& Torres-Chávez, 2014; Cardona-Londoño, Correa-Zapata, Rico-Mesa, \& Hernández-Riveros, 2013; Martínez-Ovalle, ReyesCaballero, \& González-Puín, 2013).
En el mercado actual, estos sistemas pueden implementarse de dos formas: los inyectados o conectados a red y los autónomos. Los primeros se conectan a la red eléctrica convencional para inyectar energía extra para uso residencial, de forma que en algunos países pagan esta generación extra con bonos verdes que se descuentan en el recibo de la energía eléctrica, según la normativa de cada país (Pereira-Blanco, 2015; López-Guayasamin, Castrillón, \& Cano, 2016). Los segundos corresponden a sistemas diseñados a la medida, que cumplan con la operación de cargas temporizadas predeterminadas, aun cuando no esté disponible la luz solar, permitiendo el almacenamiento de energía en baterías, quien finalmente determina la autonomía del sistema (Álvarez-López, \& Araque-Manrique, 2013; PabónFernández, Díaz-Rodríguez, \& Pardo-García, 2016).

Los Sistemas Solares Fotovoltaicos Autónomos, SFVA, a pesar de sus ventajas, presentan algunos inconvenientes, siendo el principal de ellos la falta de un sistema controlador de energía para optimizar su funcionamiento. Esta situación limita la robustez de los diseños, reduce la confianza y tranquilidad de los clientes o usuarios de estos sistemas, a la vez que dificulta el soporte técnico por parte de los vendedores de los SFVA.

Lo anterior trae como consecuencia que no se cumple con los objetivos de diseño, debido a que los usuarios conectan una mayor cantidad de cargas o dispositivos al sistema, provocando mal funcionamiento del sistema e insatisfacción de los clientes; esto último debido a que estos piensan que el sistema se descarga muy rápido y no es correcta su operación. Además, los diseñadores y vendedores de estos sistemas están insatisfechos e intranquilos, debido a las constantes llamadas de los clientes, reclamando por la no funcionalidad correcta de lo que ellos adquirieron.

En otras palabras, no existe un sistema servidor de acceso remoto para monitorear y controlar las variables del sistema SFVA de los clientes, asegurando las condiciones de operatividad $y$ 
disponibilidad, disminuyendo los tiempos en soporte técnico, los costos de transporte y el personal técnico especializado, facilitando la asistencia técnica.

Este trabajo propone profundizar en el diseño, análisis y control de un Sistema de Control Supervisor de Acceso Remoto, SCSAR, para sistemas clientes solares fotovoltaicos autónomos de 500 Watts. El documento presenta el desarrollo de un controlador para los sistemas clientes, que se encargue de cumplir con las condiciones de diseño, junto con un servidor que administre y monitoree remotamente las variables de dichos clientes.

\section{Marco teórico}

\subsection{Sistemas de control supervisor}

Desde la década de los 60 en que se lanzó al mercado el primer panel solar fotovoltaico, esta tecnología ha seguido siendo investigada y desarrollada en todo el mundo, dando lugar a mejorar la eficiencia y las ofertas en el mercado internacional de los paneles fotovoltaicos (AlonsoGarcía, Ruíz, \& Chenlo, 2006). Incluso hoy día se siguen buscando nuevas formas de hacer la tecnología fotovoltaica más eficiente, de modo que se convierta en una opción viable en cualquier escenario.

Actualmente los sistemas Tarificadores o administradores de energía para los Sistemas Solares Fotovoltaicos Autónomos, SFVA, no están definidos en el mercado nacional e incluso internacional, de modo que no existen especificaciones o estándares sobre su estructura. Con respecto a los medidores de potencia eléctrica para los SFVA, existe en el mercado un medidor de potencia real, que no lleva el consumo histórico de lo consumido por el sistema, para monitorear el consumo de cargas conectadas al mismo; es el caso del G.T. POWER Rc 130A a 60 VDC MAX, diseñado y distribuido por Aquito Solar (2012).
En la revisión de 2008 de la norma ANSI C37.1 [ANSI87], se define un sistema de administración de energía, EMS por sus siglas en inglés, denominado genéricamente sistema de supervisión. EI EMS consiste en el conjunto de elementos de control, indicadores y equipos de telemedidas asociados a la estación principal o centro de control, así como todos los dispositivos complementarios que se encuentren tanto en las subestaciones, como en las estaciones remotas.

En el campo de control remoto de redes eléctricas, se destaca el trabajo de Escudero y Luque (1994), quienes indican que los sistemas de control en redes eléctricas permiten llevar a cabo muchas tareas de manera automatizada en la generación, trasmisión y distribución de energías sobre áreas geográficas muy grandes. Entre las tareas se encuentran: el despacho de energía, la resolución de problemas producidos por restricciones de carga o caídas de tensión, el cálculo de las provisiones de demandas de la red, por mencionar algunas. Una alternativa interesante para el telecontrol de redes lo constituye la arquitectura cliente/servidor, la cual es una opción tecnológica que brinda solución al suministro de información a los usuarios; esto último permite incrementar la productividad, a través del suministro de información cuándo, cómo y dónde se necesita (Medina, 2004; Durán-Acevedo, \& Iturriago, 2012).

Entre las desventajas del modelo cliente/servidor se destaca su alto costo, debido a la complejidad técnica del servidor. Adicionalmente, el servidor es el único eslabón débil en la red ya que toda la red está construida en torno a él; para mitigar esta situación existen soluciones con sistemas de redundancia cíclica, basado en servidor, del cual se puede hacer uso y así aumentar la confiabilidad del sistema.

Los sistemas de control supervisor hacen parte de los sistemas SCADA, Supervisory Control And Data Acquisition, y aplican una estrategia de control encargada de vigilar los procesos de la planta a través de una serie de computadores, 
equipos electrónicos, sensores y actuadores interconectados, que permiten mantener las condiciones necesarias para que no ocurra ninguna perturbación en los procesos de la misma, ver figura 1.

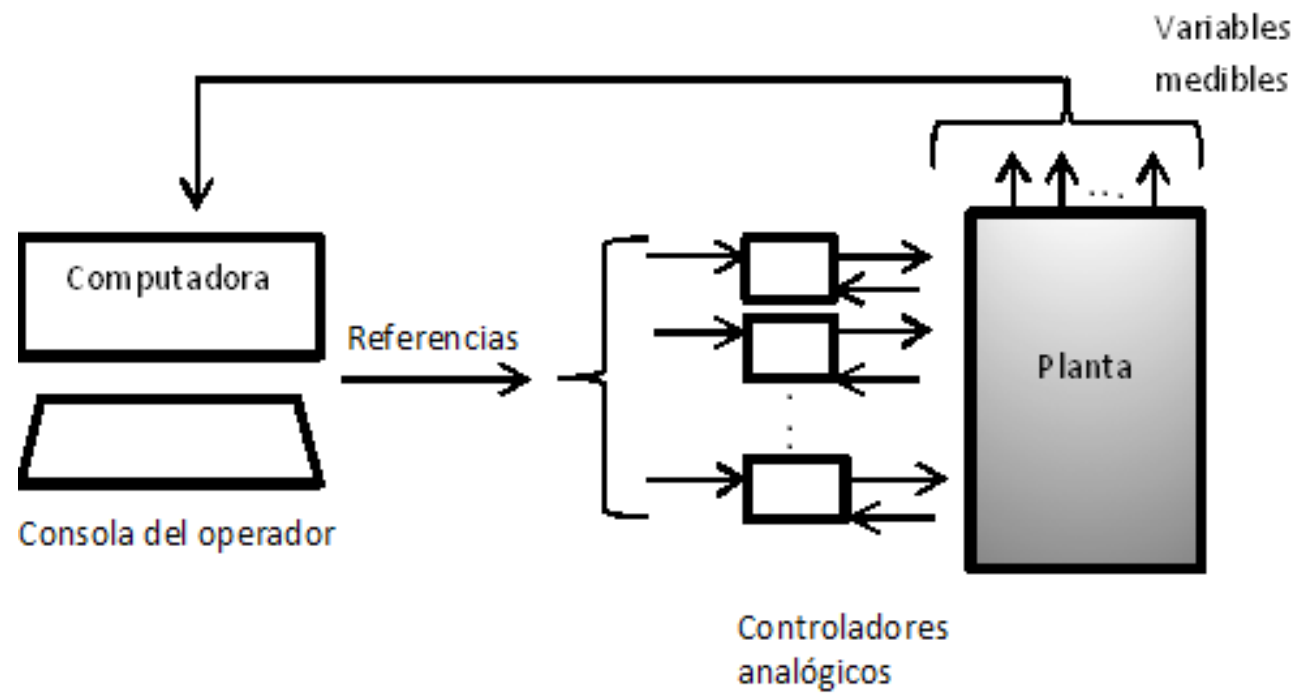

Figura 1. Esquema de un sistema de control supervisor (Martínez, 2008).

Mediante la teoría de Control Supervisor se pueden modelar los procesos de la planta, a partir de una serie de eventos y acciones que ocurren durante determinado tiempo, y que hacen posible localizar los estados donde es factible que el sistema presente bloqueos, o situaciones donde no se cumpla con la dinámica (Moody, \& Antsaklis, 1998; Music, \& Matko, 1998; Parra, Colina, \& Chacón, 2008).

\subsection{Bloques funcionales del sistema}

El sistema propuesto cuenta con dos partes o momentos fundamentales, el cliente y el servidor, las cuales se interconectan por medio de la Red de datos. El sistema cliente se encarga de ejercer el control supervisor del SFVA, para administrar la energía consumida por los usuarios. El sistema servidor se encarga de Monitorear las variables y controlar las funciones de los sistemas clientes, a través de la red de datos (Sánchez-Dams, 2013). El sistema propuesto cuenta con los bloques funcionales mostrados en la figura 2.

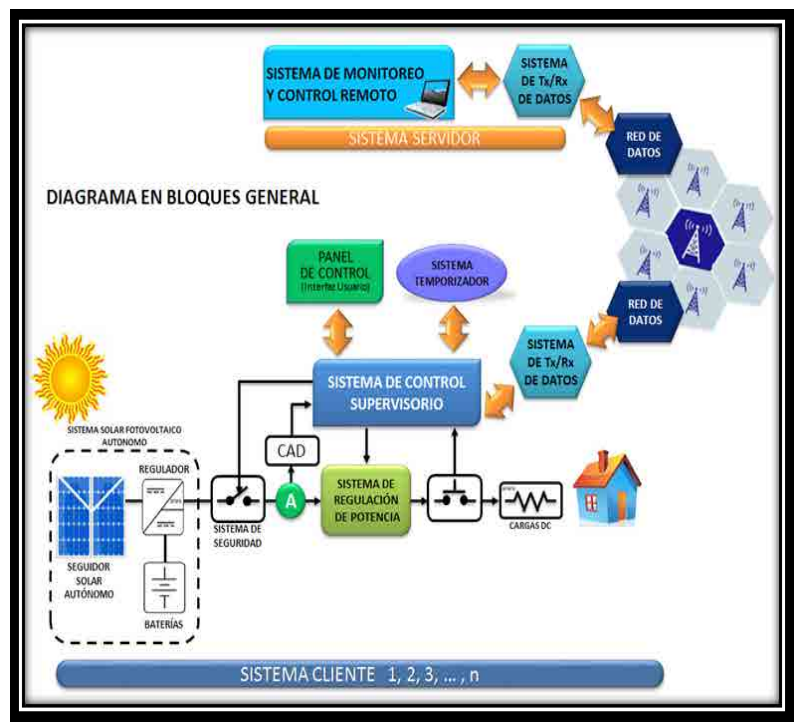

Figura 2. Diagrama en Bloques del Sistema. 
En la figura 2 se muestra como entrada al proceso un Sistema SFVA tradicional con el Panel Solar, quien es la fuente de generación de energía, seguido de un Regulador de Voltaje quien regula el voltaje a 12 VDC y las Baterías que son las que determinan la Autonomía del Sistema, funcionando bajo condiciones específicas de diseño (Beleño-Sáenz et al., 2011). De allí se conecta directamente al sistema de seguridad compuesto por un totalizador o interruptor general quien se encarga de proteger y/o totalizar al sistema por mala operación y/o condiciones adversas de trabajo. Sigue el módulo de Medición, formado por sensores de corriente, adaptadores de señal y Convertidores Análogo/Digital, CAD, quien se encarga de tomar los datos de corriente total consumida por el usuario final.

El Sistema de Control Supervisor se encarga de medir y administrar la energía consumida por los usuarios delSFVA, deforma tal quees el responsable directo de hacer cumplir la función principal para el cual fue diseñado el sistema, brindando al usuario final la confianza y disponibilidad necesaria para su correcta operación. Este, a su vez, tendrá tres funciones principales: i) Cronometrar el tiempo de conexión y desconexión por medio del sistema temporizador o tarificador de energía, de manera que, si el usuario excede el tiempo de conexión autorizado por la carga y por día, este bloqueará la salida por sobre tiempo de conexión, hasta el día siguiente, permitiéndole al usuario utilizar la energía contratada por día en otra carga, para brindarle la autonomía de diseño al sistema; ii) Administrar la corriente consumida por cada dispositivo o carga autorizado por el diseñador, de tal forma que, si el usuario conecta otro dispositivo de mayor consumo, este bloqueará la salida incidida, por sobre corriente hasta el día siguiente. iii) Desactivación por bajo nivel de la batería, debido a que es importante mantener el nivel de carga mínimo recomendado por el fabricante.

El sistema de regulación de potencia permitirá conectar y/o desconectar las cargas por medio del sistema de control Supervisor, es decir, gestionar las cargas, bloqueando o desbloqueando la que corresponda, en función de los requerimientos de diseño y usos que el usuario de al sistema SFVA(Beleño-Sáenz et al., 2011). Asimismo, el módulo de interfaz o panel de control dé usuario, deberá mostrar las variables de consumo de potencia consumida, almacenada y su estado actual.

Por medio del Sistema electrónico de Trasmisión y Recepción de datos, $T x / R x$, se envía y recibe información entre los Clientes y el Servidor, manteniendo una comunicación cerrada y segura entre los mismos, de forma que se pueda realizar el monitoreo constante y necesario por los administradores de los sistemas clientes.

El sistema de monitoreo y control tiene dos funciones principales: i) Monitorear por medio de la interfaz del Panel de Control, la potencia consumida por los sistemas clientes. ii) Controlar la potencia consumida por los sistemas clientes, permitiendo opciones de administrador para bloquear o desbloquear las salidas con excesos de consumo presentado.

En este sentido, desde el servidor del sistema de monitoreo y control remoto, se podrán monitorear y controlar manualmente las variables de los sistemas clientes; esto se realiza por medio de una interfaz o panel de control, al cual tiene acceso el administrador general del sistema, Admin, y es posible gracias al sistema de transmisión/ recepción, Tx/Rx de datos.

\section{Diseño del control supervisor}

Así las cosas, y partiendo de diseños anteriores, se hace uso de una investigación aplicada exploratoria, netamente experimental, teniendo en cuenta que los sistemas estudiados obedecen tanto a análisis teóricos como prácticos, donde a partir de la observación, medición e inferencia de principios, se identificaron y definieron las variables de entrada y salida, involucradas en el proceso de los SFVA, con el propósito de aplicar la 
estrategia de control supervisor para dar respuesta a la problemática presentada.

Para tal propósito se toma la entrada del sistema solar autónomo, a partir de la conexión del regulador de carga y el sistema de seguridad del Controlador Supervisor, para poder protegerlo. Seguidamente el sistema cuenta con un Sensor de Corriente totalizador para el sistema de carga, de forma que por medio de un CAD y una lógica especial de conexión, se pueda saber el exceso de corriente en cada conexión para cada una de las cargas o de todas a la vez.

\subsection{Condiciones de diseño}

El sistema cuenta con tres cargas de prueba a controlar: 1 Bombilla, 1 Televisor y 1 Cargador USB, que se controlarán con Relés de Estado Sólido, RES, junto con Pulsadores de control para iniciar o detener la conexión de las cargas. El Sistema de Control Supervisor cuenta con un módulo de temporización, enlazados a tres (3) contadores para determinar el tiempo y la corriente máxima por carga, cuyas condiciones no pueden superar las capacidades de diseño indicadas en la tabla 1.

\begin{tabular}{llcc}
\hline & Corrientes & & Tiempo \\
\hline Carga & Descripción & I máx (A) & t máx (h) \\
carga 1 & Bombilla & 1 & 2 \\
carga 2 & Televisor & 2 & 4 \\
carga 3 & Cargador & 2 & 6 \\
\hline
\end{tabular}

Tabla 1. Condiciones de tiempo y corriente de las cargas a controlar.

Se infiere de lo anterior que el tiempo de activación y la corriente máxima consumida de cualquier carga no podrá superar las capacidades de diseño indicadas. En este caso no se puede conectar un televisor, carga 2, que consuma más de $2 \mathrm{~A}$, lo que impediría la conexión de un televisor u otro equipo con más de esta capacidad; el tiempo máximo de 4 horas indica las horas en que puede estar encendida dicha carga, desconectando cuando este tiempo se cumpla. Se debe tener en cuenta que la corriente resultante que no sea utilizada por cada carga, se podrá distribuir en las otras equitativamente si hay demanda de consumo, para dar un buen uso de la energía al sistema solar autónomo. Cada evento del proceso va siendo registrado en el sistema cliente en segundo plano, y luego se envía al servidor para hacer el monitoreo de los mismos.

\subsection{Agente de control supervisor del sistema}

El agente de control supervisor fue desarrollado a través de autómatas de estado finito, donde se definieron las máquinas elementales de cada componente del sistema; es así para las cargas 1, 2 y 3.

En la tabla 2 se presentan la cantidad de estados y transiciones asociados a los elementos relacionados en la tabla 1. Para este caso en que solo hay tres (3) cargas modelo, se pueden acoplar ocho (8) estados resultantes de los elementos involucrados, representados con su transición básica, para comunicarse de un lado a otro.

\begin{tabular}{lcc}
\hline \multicolumn{1}{c}{ Elemento } & $\begin{array}{c}\text { No de } \\
\text { Estados }\end{array}$ & $\begin{array}{c}\text { No de } \\
\text { Transiciones }\end{array}$ \\
\hline Carga 1 (CO) & 2 & 2 \\
Carga 2 (C1) & 2 & 2 \\
Carga 3 (C2) & 2 & 2 \\
No de Estados & & \\
Resultantes & 8 & \\
\hline
\end{tabular}

Tabla 2. Autónomas del sistema: Elementos del sistema tomados para el análisis.

\subsection{Diagrama de estados de los componentes}

La representación en diagrama de estados de cada componente, ver figura 3 , indica la condición a la que se refiere cada estado; por ejemplo, para la carga 1 el estado cero (0), corresponde a la condición de carga apagada o inactiva y el estado uno (1), corresponde a la carga encendida o activa. De igual manera se representan las transiciones 
que corresponden a las cargas (C1, C2 y C3) para pasar del estado apagado a encendido (Uno (1)) y para pasar de estado abierto a cerrado (cero (0)). El mismo análisis se puede hacer para los demás componentes del sistema.

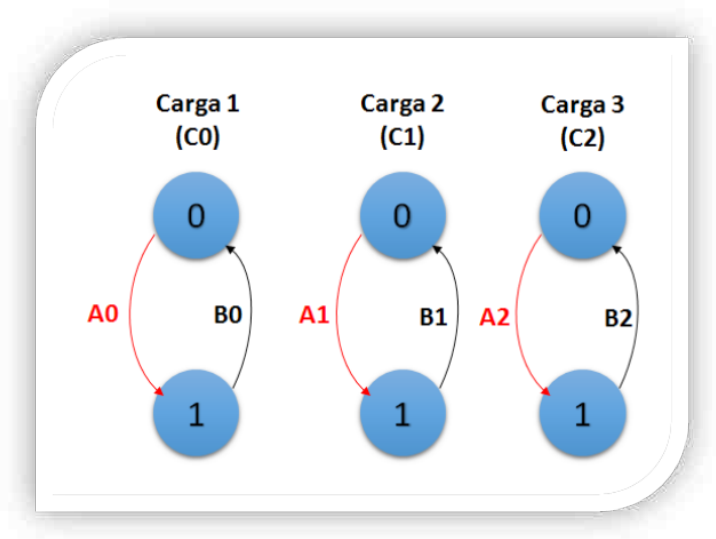

Figura 3. Representación de los elementos y estados que componen el sistema.

Esto conlleva a plantear las condiciones de funcionamiento para cada elemento finito del sistema, con el objetivo de identificar las relaciones existentes entre ellos y el proceso. Extrapolando estas condiciones al tratamiento de la autonomía del SFVA, se realiza un análisis funcional, que permite ampliar la interpretación del sistema por parte del usuario.

3.4 Análisis de los estados lógicos de los autómatas
Partiendo de lo anterior, se determina la relación lógica entre las salida controlada por donde debe fluir un máximo de corriente o Switch (SW1, SW 2 y SW 3), y el nivel sensado por el Sensor de corriente (SI), en caso tal que cuando el sistema está en 000 , indica que no hay carga conectada, y cuando está en 111 están conectadas a nivel máximo las cargas, como se indicca en la tabla 3.

\begin{tabular}{|c|c|c|c|c|c|}
\hline \multicolumn{6}{|c|}{ Medicion de Corriente con un solo AMPERIMETRO } \\
\hline & RELACIÓN & SW2 & SW1 & SWO & $\begin{array}{c}\text { I Max } \\
{[\mathrm{A}]}\end{array}$ \\
\hline $\mathrm{SI}$ & 000 & $\mathbf{0}$ & $\mathbf{0}$ & $\mathbf{0}$ & $\mathbf{0}$ \\
\hline $\mathrm{SI}$ & 001 & $\mathbf{0}$ & $\mathbf{0}$ & $\mathbf{1}$ & $\mathbf{1}$ \\
\hline $\mathrm{SI}$ & 010 & $\mathbf{0}$ & $\mathbf{1}$ & $\mathbf{0}$ & $\mathbf{2}$ \\
\hline $\mathrm{SI}$ & 011 & $\mathbf{0}$ & $\mathbf{1}$ & $\mathbf{1}$ & $\mathbf{3}$ \\
\hline $\mathrm{SI}$ & 100 & $\mathbf{1}$ & $\mathbf{0}$ & $\mathbf{0}$ & $\mathbf{3}$ \\
\hline $\mathrm{SI}$ & 101 & $\mathbf{1}$ & $\mathbf{0}$ & $\mathbf{1}$ & $\mathbf{4}$ \\
\hline $\mathrm{SI}$ & 110 & $\mathbf{1}$ & $\mathbf{1}$ & $\mathbf{0}$ & $\mathbf{5}$ \\
\hline $\mathrm{SI}$ & 111 & $\mathbf{1}$ & $\mathbf{1}$ & $\mathbf{1}$ & $\mathbf{6}$ \\
\hline
\end{tabular}

Tabla 3. Análisis de los estados lógicos del sistema.

Teniendo en cuenta todas las consideraciones anteriores, se prosigue realizando el análisis de los estados lógicos que intervienen en cada autómata del proceso y a su vez para todo el sistema. De esta forma se representan las ocho (8) condiciones posibles, dadas por las combinaciones de los estados, cambiantes de uno a otro, y en sentido contrario, así como se indica en la tabla 4.

\begin{tabular}{|c|c|c|c|c|c|c|c|c|}
\hline \multicolumn{9}{|c|}{ Análisis de los Estados Lógicos de los Autómatas } \\
\hline & 000 & $\infty 01$ & 010 & 011 & 100 & 101 & 110 & 111 \\
\hline$\infty 00$ & $\varepsilon$ & $\mathrm{CO}, \mathrm{BO}$ & $\mathrm{C} 1, \mathrm{~B} 1$ & $\infty, \mathrm{Cl}, \mathrm{BO}, \mathrm{B} 1$ & $\mathrm{C} 2, \mathrm{~B} 2$ & $\mathrm{CQ}, \mathrm{C} 2, \mathrm{BO}, \mathrm{B} 2$ & $\mathrm{C} 1, \mathrm{C} 2, \mathrm{~B} 1, \mathrm{~B} 2$ & $\mathrm{CO}, \mathrm{C} 1, \mathrm{C} 2, \mathrm{BO}, \mathrm{B} 1, \mathrm{~B} 2$ \\
\hline$\infty 01$ & BO & $\varepsilon$ & $\mathrm{C} 1, \mathrm{BO}$ & $\mathrm{C} 1$ & $\mathrm{C} 2, \mathrm{BO}$ & $\mathrm{C} 2$ & $\mathrm{C} 1, \mathrm{C} 2, \mathrm{BO}$ & $\mathrm{C} 1, \mathrm{C} 2$ \\
\hline 010 & B1 & $\mathrm{C} 1, \mathrm{BO}$ & $\varepsilon$ & co & $\mathrm{C} 2, \mathrm{~B} 1$ & $\mathrm{CO}, \mathrm{C} 2, \mathrm{~B} 1$ & $\mathrm{C} 2$ & $\mathrm{CO}, \mathrm{C} 2$ \\
\hline 011 & $B 0, B 1$ & $B 1$ & $\mathrm{BO}$ & $\varepsilon$ & $\mathrm{C} 2, \mathrm{BO}, \mathrm{B} 1$ & $\mathrm{C} 2, \mathrm{~B} 1$ & $\mathrm{C} 2, \mathrm{BO}$ & $\mathrm{C} 2$ \\
\hline 100 & B2 & $\mathrm{C} 2, \mathrm{BO}$ & $\mathrm{C} 2, \mathrm{~B} 1$ & $\mathrm{CO}, \mathrm{C} 1, \mathrm{~B} 2$ & $\varepsilon$ & $\mathrm{CQ}, \mathrm{B} 2$ & $\mathrm{C} 1$ & $\mathrm{CO}, \mathrm{C1}$ \\
\hline 101 & $\mathrm{BO}, \mathrm{B} 2$ & $B 2$ & $\mathrm{C} 1, \mathrm{BO}, \mathrm{B2}$ & $\mathrm{C} 1, \mathrm{~B} 2$ & $\mathrm{BO}$ & $\varepsilon$ & $\mathrm{C} 1, \mathrm{BO}$ & $\mathrm{C} 1$ \\
\hline 110 & $\mathrm{~B} 1, \mathrm{~B} 2$ & $\infty, B 1, B 2$ & $B 2$ & $\infty, B 2$ & B1 & $C Q, B 1$ & $\varepsilon$ & $\mathrm{CO}$ \\
\hline 111 & $\mathrm{BO}, \mathrm{B} 1, \mathrm{~B} 2$ & $\mathrm{~B} 1, \mathrm{~B} 2$ & $\mathrm{BO}, \mathrm{B} 2$ & $B 2$ & $\mathrm{BO}, \mathrm{B} 1$ & $B 1$ & $\mathrm{BO}$ & $\varepsilon$ \\
\hline
\end{tabular}


Tabla 4. Análisis de los estados lógicos del sistema.

En esta representación se muestra el cambio de estado entre una condición de carga para volver a su estado nominal o normal, teniendo en cuenta que son tres (3) estados y tres (3) cargas. En este caso se debe tener en cuenta lo indicado anteriormente para las nemotécnicas usadas.

\subsection{Autómata global, luego de la composición}

Luego de tener asegurada la representación de las condiciones de entradas o salidas, tabla 3, que corresponde al análisis de estados lógicos, se procede a realizar una composición aproximada del diagrama general de los autómatas para el sistema de control supervisor, ver figura 4.

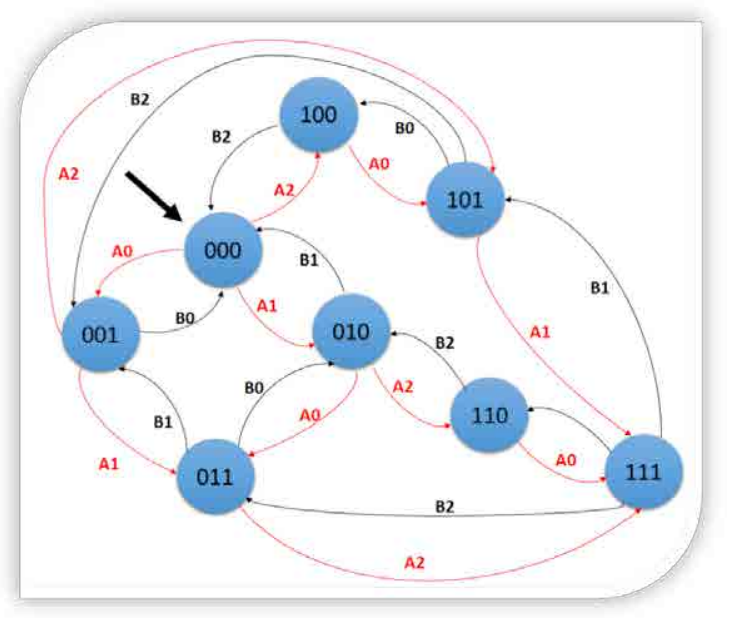

Figura 4. Representación global de los autómatas del sistema.

En el diagrama de la figura 4 se reflejan todas las condiciones que podrían suceder para que el sistema de control supervisor responda de manera positiva a las transiciones de estados que se efectúen en el mismo. Estas representaciones se globalizaron teniendo en cuenta todos los principios del control supervisor.

\subsection{Supervisor mínimamente restrictivo}

Para reducir aún más el número de estados y transiciones, es necesario definir un número de restricciones que permitan establecer el correcto funcionamiento del sistema. Este sistema supervisor define las restricciones de los SFVA o los estados posibles que deben cumplirse. El lenguaje físicamente posible define las acciones que puede tomar el sistema. Para el análisis del sistema, se tomará el caso particular de funcionamiento establecido por el operador o lenguaje posible, el cual define el lenguaje del sistema.

A pesar de las restricciones, este modelo permite ilustrar claramente la idea de funcionalidad del sistema, para poder implementar el control, debido a las zonas de no interconexión donde se presentan dichos sistemas de forma ideal, segura y factible, teniendo en cuenta para ello los costos de implementación. En este caso sería por medio de la red telefónica Móvil GSM, para poder enviar los comandos a la central de operación.

Lo anterior permite decir que, debido a la criticidad de uso de cada variable a medir, no se depende directamente de una secuencia lógica como tal, de forma que no podemos definir un esquema supervisor mínimamente restrictivo. Pero se puede mostrar un diagrama mínimo, ver figura 5, donde se notan los valores para cada sensor y su prioridad para tener en cuenta y conocer el estado de carga en el proceso.

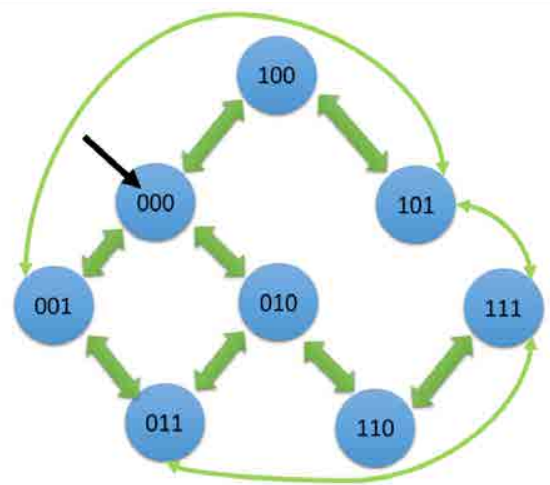


Figura 5. Representación global de los autómatas del sistema final.

\subsection{Simulación del autómata supervisor}

Se procede a llevar el autómata global de la figura 5, con sus estados marcados, al proceso de simulación desde la plataforma JFLAP, Versión 7.0 (2009), sin presentarle ninguna reducción de datos por verificación, quedando como se ilustra en la figura 6.

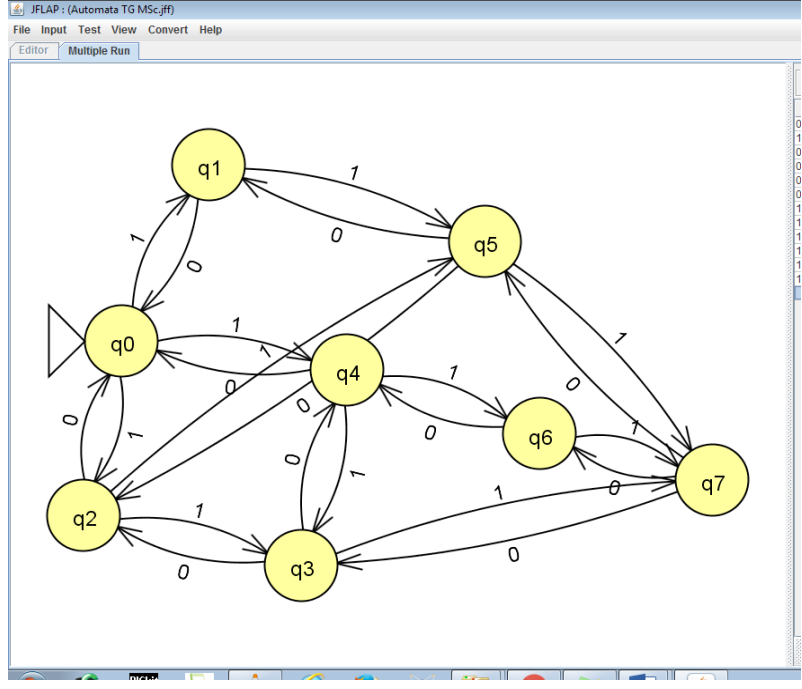

Figura 6. Simulación del autómata global del supervisor.

Así mismo se simulan todos los estados anteriores del autómata programado, para llevarlo a un programa, software o firmware y observar el comportamiento real; esto se muestra en la figura 7.

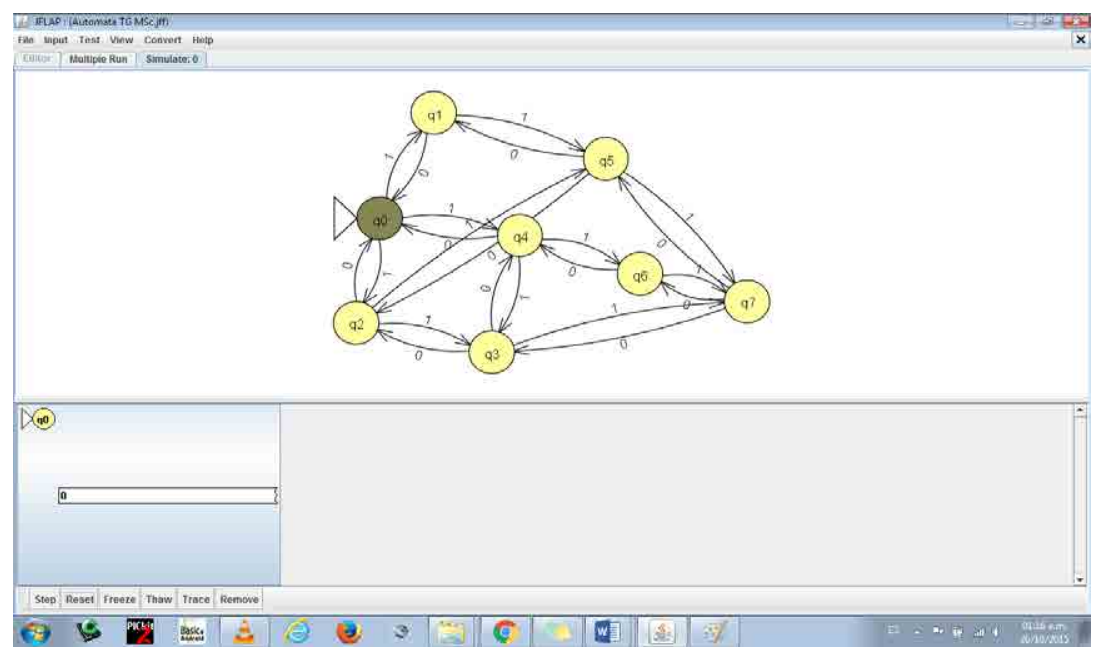

Figura 7. Pruebas simuladas del autómata global.

A través de la prueba mencionada anteriormente, se pudo constatar que las condiciones de diseño del sistema de control supervisor quedan validadas para proceder a su implementación, debido a que simula de manera adecuada para cada una de las entradas especificadas.

Es decir, con la información disponible en este punto se brinda una solución al diseño en cuanto a: 
la comunicación, la trasmisión recepción de datos al servidor y la parte del control Supervisor. Como dispositivo controlador se sugiere a los PIC de gama alta, p.ej. el PIC18F4520, microcontroladores que han sido empleados ampliamente en aplicaciones de instrumentación y control de procesos, gracias a su gran versatilidad (Fernández-Morales, \& Duarte, 2012; Cárdenas, \& Prieto-Ortiz, 2015; Torres-Barahona, León-Medina, \& Torres-Díaz, 2012; León-Medina, \& Torres-Barahona, 2016).

Finalmente, se puede decir que la metodología de diseño permitió la propuesta de un sistema para el monitoreo y control remoto de los SFVA, orientado al diagnóstico a distancia de todos los elementos del mismo, para disminuir los costos en transporte y mantenimiento y personal técnico. El sistema de control impedirá que los usuarios superen las condiciones máximas de tiempo y consumo de energía establecidas por el diseñador del SFVA, reduciendo las quejas por el supuesto mal funcionamiento que los clientes de este tipo de sistemas suele presentar.

\section{Conclusiones}

El sistema aquí propuesto está desarrollado bajo el enfoque de la técnica de control supervisor, con la unión de nuevas tecnologías de software y hardware, incluyendo las telecomunicaciones, de forma que aplica nuevas tendencias de personalización al usuario y comunicación de última tecnología.

La metodología aplicada permite deducir que el análisis de criticidad del proceso es una estrategia adecuada para implementar el control supervisor en Sistemas SFVA ubicados en Zonas No interconectadas. El sistema controlador resultante cumple la función de medir y administrar la energía consumida por los usuarios del SFVA, manejando para ello tres variables principales: el tiempo de conexión y desconexión por carga y por día, autorizados al usuario; la corriente consumida por cada dispositivo o carga autorizado por el diseñador; el nivel de carga de la batería para mantenerlo por encima del mínimo permitido para su correcta operación.

En síntesis, el sistema propuesto realiza un Aporte relacionado con la versatilidad que se brinda al SFVA, en cuanto a la administración de la potencia energética consumida por el usuario. Adicionalmente, esta solución podría extenderse a otros sistemas de generación, ya sean estos solares, eólicos o cualquier otro sistema de energía renovable que requiera control y supervisión del consumo.

\section{Referencias}

Alonso-García, M. C., Ruíz, M., \& Chenlo, F. (2006). Experimental study of mismatch and shading effects in the I-V characteristics of a photovoltaic module. Solar Energy Materials and Solar Cells, 90 (3), 329-340.

Álvarez-López, R. A., \& Arango-Manrique, A. (2013). Implementación de la generación fotovoltaica como respaldo ante fallos en la red de distribución, Revista Colombiana de Tecnologías de Avanzada, 2 (22), 14-19.

American National Estándar Institute, ANSI. (2008). ANSI C37.1 [ANSI-87], última edición. Recuperado de: www.ansi.org.

Aquito Solar (2012). Medidor de Energía Solar. Recuperado de: http://www.aquitosolar.cl/ medidores-energia-solar/185-PWM130AMI.html

Beleño-Sáenz, K., Pardo-García, V., \& TorresChávez, I. (2013). Control aplicado a una estructura mecánica para el posicionamiento automático de módulos fotovoltaicos, Revista Colombiana de Tecnologías de Avanzada, 2 (22), 138-144.

Beleño-Sáenz, K., Torres-Chávez, I., Pardo-García, A., \& Díaz-Rodríguez, J. L. (2011). Sistema de posicionamiento solar utilizando la plataforma de Labview y el microcontrolador PIC18F4550, 
Revista Colombiana de Tecnologías de Avanzada, 2 (18), 125-131.

Cárdenas, J. A., \& Prieto-Ortíz, F. A. (2015). Diseño de un algoritmo de corrección automática de posición para el proceso de perforado PCB, empleando técnicas de visión artificial. Revista de Investigación, Desarrollo e Innovación, 5 (2), 107118. doi: $10.19053 / 20278306.3720$

Cardona-Londoño, J., Correa-Zapata, S., RicoMesa, E., \& Hernández-Riveros, J. (2013). Robot móvil solar. Revista Colombiana de Tecnologías de Avanzada, 1 (21), 10-18.

Durán-Acevedo, C. M., \& Iturriago, A. X. (2012). Automatización de un Sistema de Suministro de Agua Potable a Través de la Tecnología Zigbee. Revista Colombiana de Tecnologías de Avanzada, 2 (20), 36-42.

Escudero, J., \& Luque, J. (1994). Telecontrol de Redes Eléctricas. (Tesis). Universidad de Sevilla, Departamento de tecnología electrónica.

Fernández-Morales, F. H., \& Duarte, J. E. (2012). Desarrollo de un caudalímetro digital para la medición de caudal de ríos. Revista de Investigación, Desarrollo e Innovación, 3 (1), 44-51. Recuperado de: http://revistas.uptc.edu.co/revistas/index.php/ investigacion_duitama/article/view/2130

García, M. (2005). Caracterización y modelado de asociaciones de dispositivos fotovoltaicos. (Tesis doctoral). CIEMAT.

Gómez-Rojas, O., Díaz-Lagos, M., Blandón-Montes, A., \& Martínez-Ovalle, S. (2016). Presencia de elementos contaminantes como $\mathrm{Cd}, \mathrm{As}, \mathrm{Pb}$, Se y $\mathrm{Hg}$ en carbones de la zona Cundiboyacense, Colombia. Revista de Investigación, Desarrollo e Innovación, 7 (1), 141-150. doi:http://dx.doi. org/10.19053/20278306.v7.n1.2016.5604
JFLAP, Versión 7.0 (2009). Software JFLAP. Recuperado de: http://www.jflap.org/jflaptmp/

Leal-González, F. A., Hernández-Cely, M. M. (2014). Estudio del potencial eólico y solar de Cúcuta, Norte de Santander. Revista Colombiana de Tecnologías de Avanzada, 1 (23), 142-147.

León-Medina, J. X., \& Torres-Barahona, E. A. (2016). Herramienta para el diseño de sistemas de posicionamiento tridimensional usados en fabricación digital. Revista de Investigación, Desarrollo e Innovación, 6 (2), 155-167. doi: http:// doi.org/10.19053/20278306.4603

López-Guayasamin, M. R., Castrillón, O. D. \& Cano, E. (2016). Análisis de eventos sobre transformadores de distribución en una empresa del sector eléctrico en Colombia. Revista Colombiana de Tecnologías de Avanzada, 1 (27), 112-117.

Martínez, G. (2008). Manual teórico, Integración de Sistemas Automáticos. Universidad tecnológica de Torreón.

Martínez-Bernal, M. S. (2013). Determinación de la productividad y competitividad de la pequeña minería del distrito minero del norte de Boyacá. Revista de Investigación, Desarrollo e Innovación, 3 (2), 72-86. doi: 10.19053/20278306.2168

Martínez-Ovalle, S., Reyes-Caballero, F., \& González-Puín, L. X. (2013). Protección radiológica a trabajadores y público en instalaciones que operan radioisótopos industriales. Revista de Investigación, Desarrollo e Innovación, 3 (2), 120124. doi: $10.19053 / 20278306.2166$

Medina, H. (2004). Impacto del Paradigma ClienteServidor en el Proceso de Selección de Personal. En (Silva-Ledesma Tejada, David: "Cliente Servidor"), Delegación CITMA Villa Clara, IX Evento Redes, Una Opción sin Límites. CITMATEL. 
Moody, J., \& Antsaklis, J. (1998). Supervisory Control of Discrete Event Systems Using Petri Nets. USA: University of Notre Dame in USA.

Music, G., \& Matko, D. (1998). Petri Net Based Supervisory Control Flexible Bacth Plants. Symposium on Large Scale Systems. 2. Rio Patras, Greece.

Pabón-Fernández, L., Díaz-Rodríguez, J., \& PardoGarcía, A. (2016). Simulación del inversor multinivel de fuente común como variador de frecuencia para motores de inducción. Revista de Investigación, Desarrollo e Innovación, 7 (1), 165-180. doi: https:// doi.org/10.19053/20278306.v7.n1.2016.5636

Parra, C., Colina, E., \& Chacon, E. (2008). Intelligent Supervisory Control of Continuous Processes Exposed to Failures. Revista Colombiana de Tecnologías de Avanzada, 2 (12), 39-46.

Pereira-Blanco, M. J. (2015). Relación entre energía, medio ambiente y desarrollo económico a partir del análisis jurídico de las energías renovables en Colombia. Saber, Ciencia y Libertad, 10 (1), 35-60. doi:http://dx.doi.org/10.22525/ sabcliber.2015v10n1.3660

Reyes-Caballero, F., Fernández-Morales, F., \& Duarte, J. (2016). Panorama energético. Revista de Investigación, Desarrollo e Innovación, 7 (1), 151163. doi:http://dx.doi.org/10.19053/20278306. v7.n1.2016.5605

Sánchez, T., \& Escobar, R. (2009). Energía solar fotovoltaica: una opción para la electrificación rural. Lima, Perú.
Sánchez-Dams, R. D. (2013). Estado del arte del desarrollo de sistemas embebidos desde una perspectiva integrada entre el hardware y software. Revista Colombiana de Tecnologías de Avanzada, 2 (22), 98-105.

Sánchez-Molina, J., Gelves-Díaz, J. F., \& Ramírez, R. P. (2014). Implementación de un sistema tipo SCADA para mejorar los procesos de secado y cocción de la ladrillera Sigma Ltda., Revista Colombiana de Tecnologías de Avanzada, 2 (24), 80-85.

Sandoval-Ruiz, C. (2014). Adaptive Control in VHDL Applied to a Solar Oven, Revista Colombiana de Tecnologías de Avanzada, 2 (20), 80-85.

Torres-Barahona, E. A., León-Medina, J. X. \& TorresDíaz, E. (2012). Sistema de posicionamiento aplicado a la técnica de impresión 3D modelado por deposición fundida. Revista de Investigación, Desarrollo e Innovación, 3 (1), 25-32. Recuperado de: http://revistas.uptc.edu.co/revistas/index.php/ investigacion_duitama/article/view/2135/2091

Vargas, J. A., Velásquez-Clavijo, F., \& TorresGómez, C. (2016). Desarrollo del prototipo de un hidrogenerador eléctrico como alternativa de generación de energía limpia en zonas rurales. Ingeniare, Ingeniare 20, 91-101.

Vides-Herrera, C. A., Pardo-García, A., \& TorresChávez, I. (2014). Módulo robótico de seguimiento solar usando MPPT supervisado y controlado vía Ethernet. Revista Colombiana de Tecnologías de Avanzada, 1 (23), 112-119. 